\title{
Correction to: Human-centered design as a guide to intervention planning for non- communicable diseases: the BIGPIC study from Western Kenya
}

\author{
Claudia L. Leung ${ }^{1}$, Mackenzie Naert², Benjamin Andama ${ }^{3}$, Rae Dong ${ }^{2}$, David Edelman' ${ }^{1}$ Carol Horowitz², \\ Peninah Kiptoo ${ }^{3}$, Simon Manyara ${ }^{3}$, Winnie Matelong ${ }^{3}$, Esther Matini ${ }^{3}$, Violet Naanyu ${ }^{4}$, Sarah Nyariki ${ }^{3}$, \\ Sonak Pastakia ${ }^{5}$, Thomas Valente ${ }^{6}$, Valentin Fuster ${ }^{2}$, Gerald S. Bloomfield ${ }^{1}$, Jemima Kamano ${ }^{4}$ and \\ Rajesh Vedanthan ${ }^{7 *}$
}

\section{Correction to: BMC Health Serv Res (2020) 20:415 https://doi.org/10.1186/s12913-020-05199-1}

Following the publication of the original article [1], it was noted that due to a typesetting error the Figs. 1, 4, 5 and 6 are not correct.

The correct figures have been included in this correction, and the original article has been corrected.

The author affiliations need to be revised as below and the original article has been corrected.

Claudia L. Leung ${ }^{1}$, Mackenzie Naert ${ }^{2}$, Benjamin Andama $^{3}$, Rae Dong ${ }^{2}$, David Edelman ${ }^{1}$, Carol Horowitz ${ }^{2}$, Peninah Kiptoo ${ }^{3}$, Simon Manyara ${ }^{3}$, Winnie Matelong ${ }^{3}$, Esther Matini ${ }^{3}$, Violet Naanyu ${ }^{4}$, Sarah Nyariki ${ }^{3}$, Sonak Pastakia $^{5}$, Thomas Valente ${ }^{6}$, Valentin Fuster ${ }^{2}$, Gerald S. Bloomfield ${ }^{1}$, Jemima Kamano ${ }^{4}$, and Rajesh Vedanthan ${ }^{7 *}$

\section{Author details}

${ }^{1}$ Duke University Medical Center, 10 Duke Medicine Circle, Durham, NC 27710, USA. ${ }^{2}$ Division of General Internal Medicine, Duke University School of Medicine, 200 Morris St. 3rd floor, Durham, NC 27701, USA. ${ }^{3}$ Icahn School of Medicine at Mount Sinai, 1 Gustave L. Levy PI, New York, NY 10029, USA. ${ }^{4}$ Academic Model Providing Access to Healthcare (AMPATH), P.O. Box 4606, Eldoret 30100, Kenya. ${ }^{5}$ Department of Behavioral Sciences, School of Medicine, College of Health Science, Moi University College of Health

The original article can be found online at https://doi.org/10.1186/s12913020-05199-1.

* Correspondence: Rajesh.Vedanthan@nyulangone.org

${ }^{7}$ Department of Preventive Medicine, Keck School of Medicine, University of Southern California, Los Angeles, CA, USA

Full list of author information is available at the end of the article

Sciences, Eldoret, Kenya. ${ }^{6}$ Purdue University, Purdue University College of Pharmacy, Purdue-Kenya Partnership, West Lafayette, IN, PO Box 5760, Eldoret 30100, Kenya. 'Department of Preventive Medicine, Keck School of Medicine, University of Southern California, Los Angeles, CA, USA.

Published online: 12 August 2020

\section{Reference}

1. Leung $\mathrm{CL}$, et al. Human-centered design as a guide to intervention planning for non-communicable diseases: the BIGPIC study from Western Kenya. BMC Health Serv Res. 2020;20:415.

(c) The Author(s). 2020 Open Access This article is licensed under a Creative Commons Attribution 4.0 International License, which permits use, sharing, adaptation, distribution and reproduction in any medium or format, as long as you give appropriate credit to the original author(s) and the source, provide a link to the Creative Commons licence, and indicate if changes were made. The images or other third party material in this article are included in the article's Creative Commons licence, unless indicated otherwise in a credit line to the material. If material is not included in the article's Creative Commons licence and your intended use is not permitted by statutory regulation or exceeds the permitted use, you will need to obtain permission directly from the copyright holder. To view a copy of this licence, visit http://creativecommons.org/licenses/by/4.0/. The Creative Commons Public Domain Dedication waiver (http://creativecommons.org/publicdomain/zero/1.0/) applies to the data made available in this article, unless otherwise stated in a credit line to the data. 
DISCOVER. Observe, experience, and understand

STEP 1: Understanding the Community

16 FGDs

- Participant groups: microfinance (MF) group members,

clinicians, patients with NCDs across 14 communities

- Methods: semi-structured guided FGDs

5 mabaraza

- Participants: constituents of four rural communities

RESULTS:

Methods: semi-structured large group

- Barriers and

facilitators to care

discussion

- Contextual factors

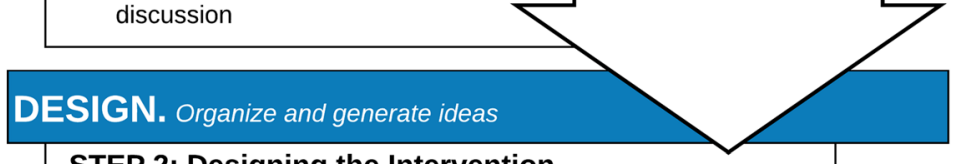

STEP 2: Designing the Intervention

Participants: A transdisciplinary team (Design Team) composed of: clinicians, CHWs, village leaders, pharmacists, nutritionist, and MF program officers

Methods: A series of meetings over six weeks to define challenges to NCD care and design an intervention model that meets the

specific needs of the local community.

RESULTS:

Prototype Model

- Monthly group care with

concurrent microfinance

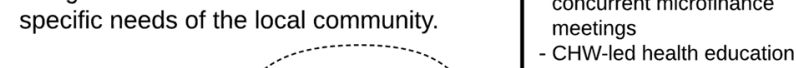

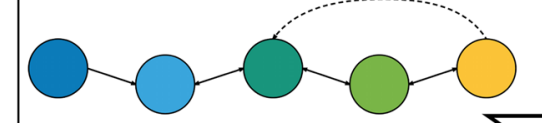

- Village-based screening

methods

- Established ideal group size

See Figure 3. Design Team Meeting

- Developed CHW and

participant training

TEST. Test and evaluate creative strategies

STEP 3a: Assess community acceptance 8 FGDs

- Participant groups: MF group members, clinicians, CHWs, patients with NCDs across seven rural communities

- Methods: Model presented to potential end-users with collection of qualitative feedback through FGDs.

\section{STEP 3b: Pilot study}

6-month community-based pilot study inclusive of:

- CHW and participant training

- Screening methods

- Monthly MF and group care meetings FGDs and key informant interviews with personnel (clinician and CHWs) and participants at 1-, 3-, and 6-months.

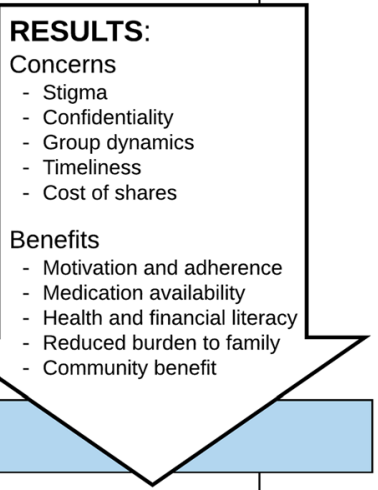

REFINE. Tailor solutions to meet client needs

STEP 4: Intervention Refinement

Participants: original Design Team, now including representatives from the pilot study

Methods: A series of meetings to review the results of qualitative feedback gathered in Step 3. Prototype model refined to create a final integrated group care and MF model (BIGPIC model).

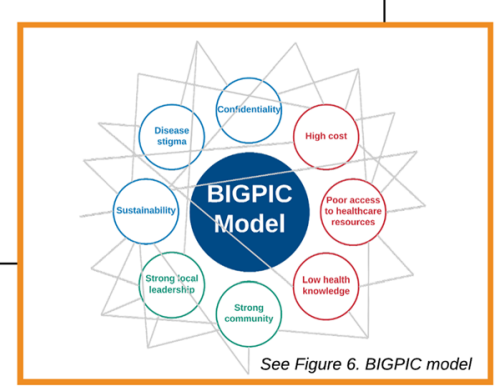

Fig. 1 Human-centered design stages and activities in the BIGPIC design process. Steps 1-4 describe each stage of our project in the context of the HCD steps (Discover, Design, Test, and Refine). As HCD is an iterative process, the arrows describe how the results of each step impact the next 


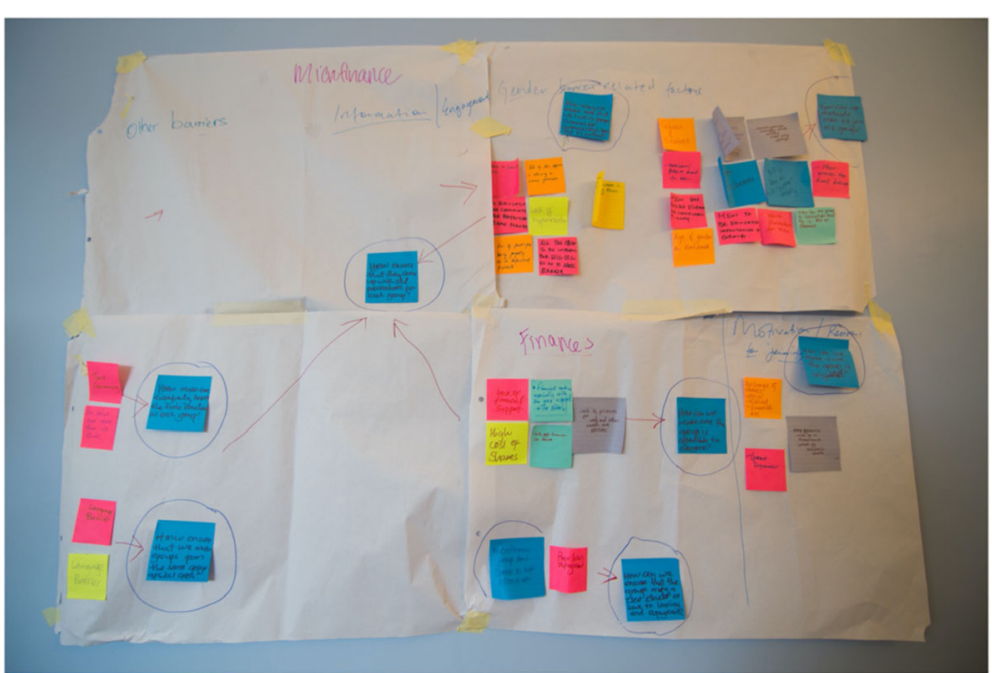

Fig. 4 Key themes were organized together to stimulate idea generation

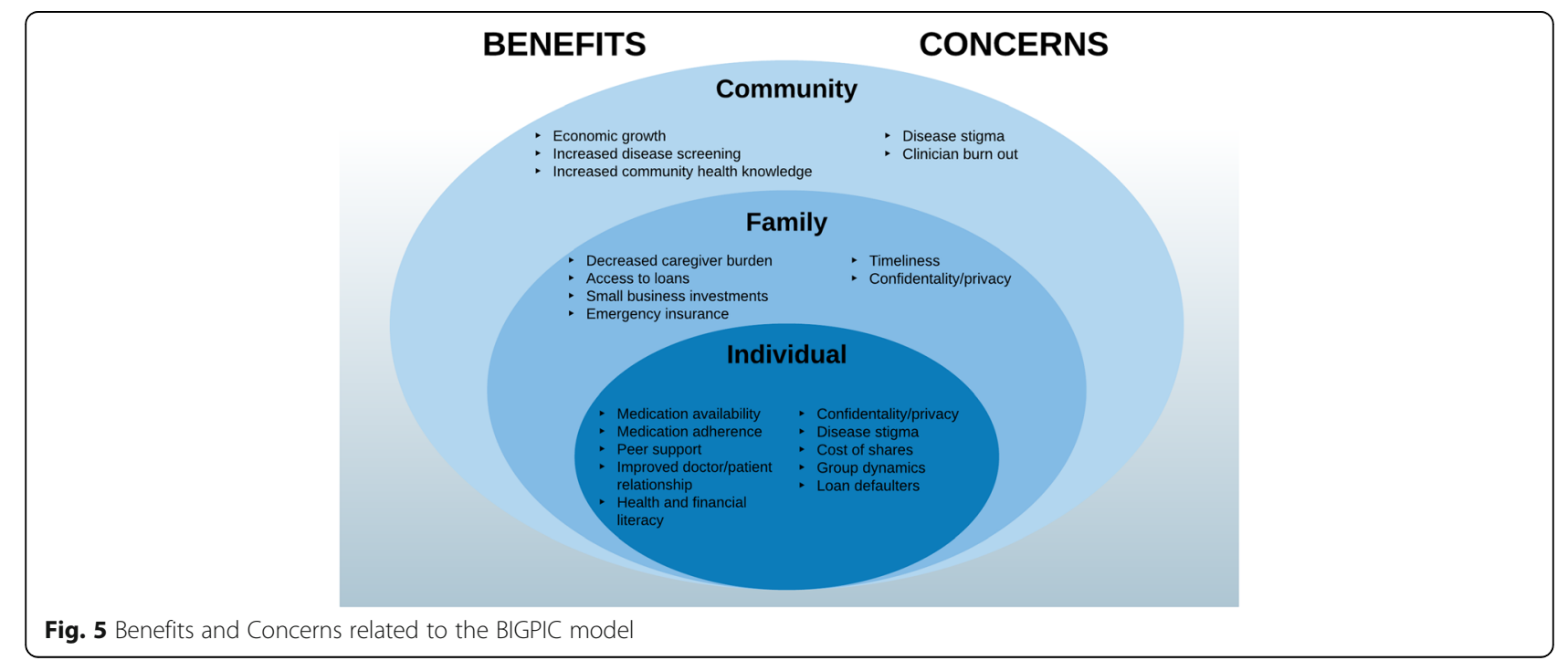




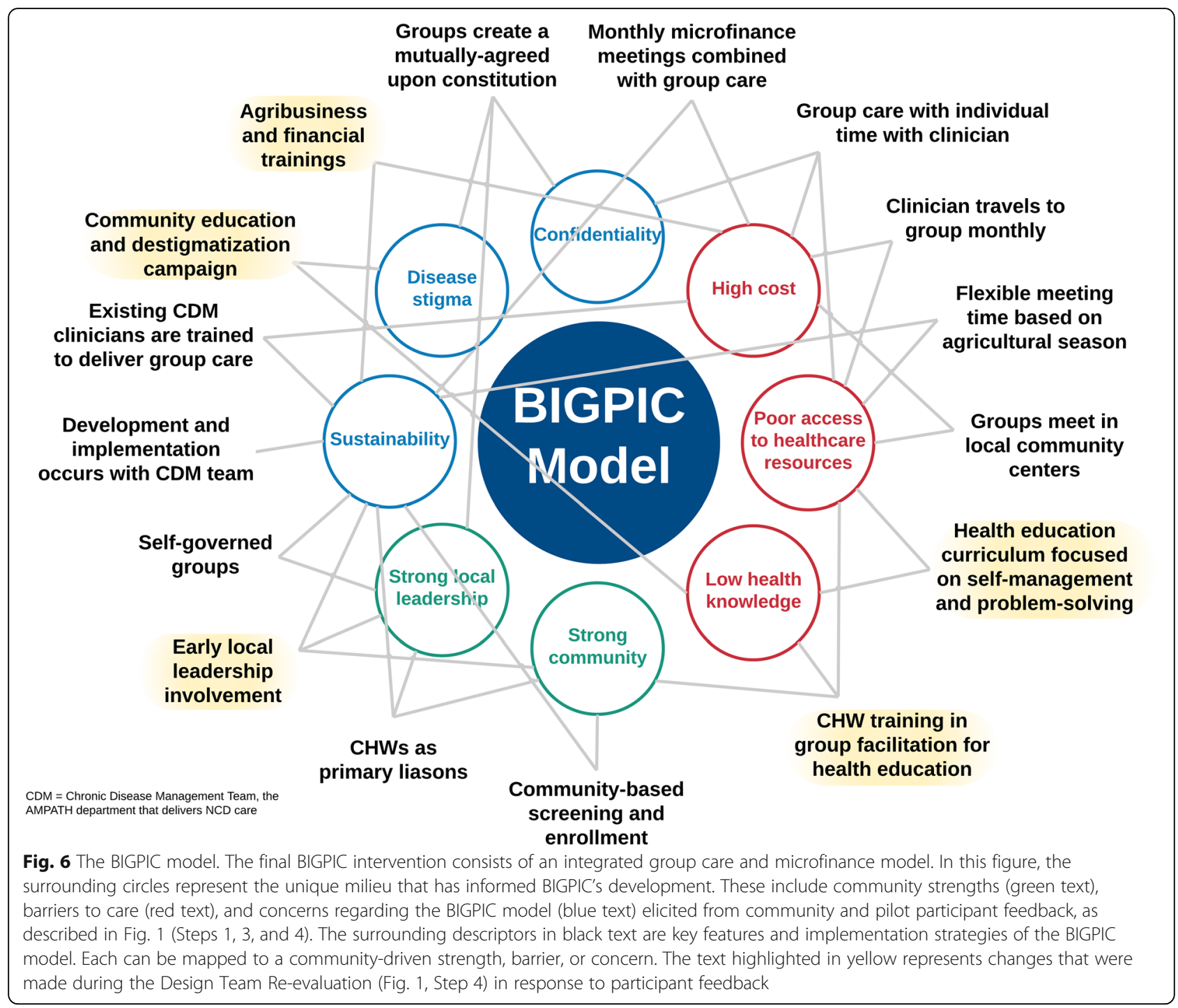

